九州大学学術情報リポジトリ

Kyushu University Institutional Repository

\title{
Measuring Load of Phosphate in the Environment by Passive Sampling Techniques : An Introduction
}

\section{Phong, Thai Khanh}

Research Fellow, National Centre for Environmental Toxicology (Entox), University of Queens land

Phuc, Dam Hoang

Hanoi University of Science and Technology

Phi, Thai Ha

University of Transport and Communications

Hiramatsu, Kazuaki

Laboratory of Water Environment Engineering, Division of Bioproduction Environmental Sciences,

Department of Agro-Environmental Sciences, Faculty of Agriculture, Kyushu University

https://doi.org/10.5109/26175

出版情報: 九州大学大学院農学研究院紀要. 58 (1)，pp.153-157，2013-02. Faculty of Agriculture， Kyushu University

バージョン :

権利関係 : 


\title{
Measuring Load of Phosphate in the Environment by Passive Sampling Techniques - An Introduction
}

\author{
Thai Khanh PHONG ${ }^{*}$, Dam Hoang PHUC ${ }^{2}$, Thai Ha PHI ${ }^{3}$ \\ and Kazuaki HIRAMATSU
}

\author{
Laboratory of Water Environment Engineering, Division of Bioproduction Environmental Sciences, \\ Department of Agro-Environmental Sciences, Faculty of Agriculture, \\ Kyushu University, Fukuoka 812-8581, Japan \\ (Received October 28, 2012 and accepted November 8, 2012)
}

\begin{abstract}
Phosphorus has a number of indispensable biochemical roles, but its natural deposition and the low solubility of phosphates as well as their rapid transformation to insoluble forms make the element commonly the growth-limiting nutrient, particularly in aquatic ecosystems. Famously, phosphorus that reaches water bodies is commonly the main cause of eutrophication. This undesirable process can severely affect many aquatic biotas in the world. More management practices are proposed but long-term monitoring of phosphorus level is necessary to ensure that the eutrophication won't occur. Passive sampling techniques, which have been developed over the last decades, could provide several advantages to the conventional sampling methods including simpler sampling devices, more cost-effective sampling campaign, providing flow proportional load as well as representative average of concentrations of phosphorus in the environment. Although some types of passive samplers are commercially available, their uses are still scarcely reported in the literature. In Japan, there is limited application of passive sampling technique to monitor phosphorus even in the field of agricultural environment. This paper aims to introduce the relatively new P-sampling techniques and their potential to use in environmental monitoring studies.
\end{abstract}

Key words: phosphate, passive sampler, environmental monitoring, water quality

\section{INTRODUCTION}

Phosphorus is a vital nutrient for converting sunlight into usable energy, and essential to cellular growth and reproduction. Under natural conditions phosphorus is typically scarce in water. However, it is now well known that anthropogenic phosphorus, especially from agricultural sources, can trigger excessive algae bloom and cause degraded water quality. Phosphorus occurs in dissolved organic and inorganic forms in the water phase or adsorbed to sediment particles. Phosphates, the inorganic form, are preferred for plant growth, but other forms can be used when phosphates are unavailable.

In order to protect the quality of the environment, it is important to carry out continuous environmental monitoring and management schemes. However, the conventional method of using spot/grab samples can be extremely laborious and expensive for a long-term monitoring. Moreover, the grab samples only indicate the state of the environment at a particular time and space. While it may be suitable for monitoring an environmental compartment in steady state, the information obtained from grab samples is less representative for actual environmental bodies which are usually in transient state, e.g. the level of phosphorus loads in rivers and streams because of quickly changing dissolved phosphorus concentrations $P(t)$ and water flow rates $Q(t)$.

Depending on its sources, riverine $P(t)$ can fluctuates

\footnotetext{
1 Research Fellow, National Centre for Environmental Toxicology (Entox), University of Queensland, Australia

2 Hanoi University of Science and Technology, Vietnam

3 University of Transport and Communications, Hanoi, Vietnam

* Corresponding author (E-mail: p.thai@uq.edu.au)
}

diurnally as well as seasonally, and in addition depends on water discharge $Q(t)$. Muller et al. (2008) has derived a equation for the load of phosphorus $\left(L_{\Delta t}\right)$ transported by the river within a given period of time $\Delta t$ as

$$
L_{\Delta t}=\int_{t 1}^{t 2} Q(t) \times P(t) d t
$$

An accurate measurement of $L_{\Delta t}$ requires continuous monitoring of both $Q(t)$ and $P(t)$. And arguably at the moment, $Q(t)$ can be measured relatively accurately in site-specific situations but no simple method is available for continuously surveying the ambient $P(t)$ concentrations.

Passive sampling can overcome such shortcoming by continuously capturing the compounds of interest in the sampled environment and providing a time-weight average concentration over the sampling (deploying) period (Kot-Wasit et al., 2007). Recent development in passive sampling techniques has delivered the possibility of measuring time-average concentration of dissolved phosphorus in the water phase by using appropriate sorbents (Müller et al., 2007, Pichette et al., 2009, Rozemeijer et al., 2010, Panther et al., 2011, de Jonge and Rothenberg, 2005).

Meanwhile, the application of passive sampling in Japan is still very limited, especially in the area of aquatic environmental monitoring. In the previous sister paper (Phong et al., 2012), we have briefly reviewed the use of passive samplers for a range of organic pollutants such as pesticides, veterinary drugs and the situation in Japan. Similarly, in this paper, we would like to introduce the approach of passive sampling in the newer area of measuring ionic and inorganic compounds where there are 
many potentials but the application is still scarcely reported, especially in Japan. The aims of this paper are to:

- Introduce the passive sampling techniques that are currently in use in the world to monitor phosphorus

- Description of some potential applications of these passive sampling techniques

\section{PASSIVE SAMPLING TECHNIQUES FOR PHOSPHORUS IN THE ENVIRONMENT}

\section{General introduction}

The passive sampling system is based on the Fick's Law, and relies on movement (due to partition or adsorption) of compounds of interest from the sampled medium to the collecting medium (Górecki and Namieśnik, 2002). Over decades since their conception, passive samplers have been deployed in various environments such as freshwater, effluents and marine environments to monitor a range of pollutants. In general, the amount of the sampled compounds depends on the deployment time and their concentrations in the sampled medium, but some environmental conditions $(\mathrm{pH}$, temperature, turbulence) may affect the kinetics of the process. The concentration of the target compounds is obtained as a timeweighted average (TWA), representing the average concentration of the analytes in the sampled medium during the exposure time (Górecki and Namieśnik, 2002).

Phosphate concentrations in environmental compartments $\left(C_{w}\right)$ can be determined from the mass $\left(M_{s}\right)$ of phosphate accumulated within the deployed passive sampler, assuming that the sampler is operating in the linear or integrative mode

$$
C_{w}=\frac{M_{s}}{R_{s} t}
$$

where $t$ is the deployment time, and $R_{s}$ is the sampling rate.

Values of $R_{\mathrm{s}}$ may depend on exposure conditions such as flow rate, ionic strength, and temperature (O'Brien et al., 2011). The following techniques are designed to measuring the mass $M_{s}$ in order to estimate a time-average concentration of phosphate in this environment.

The use of ferrihydrite, a ferric oxyhydroxide mineral, as a sequestering phase has led to the development of two passive sampling methods for the measurement of phosphate concentration in water as presented below. The first and earlier method employs ferrihydrite within a diffusive gradient in thin-film (DGT) gel (Zhang et al., 1998). A more recent method, called the P-trap, contains a ferrihydrite suspension which is in contact with the outside environment through a commercially available filter membrane (Müller et al., 2007)

The last and newly proposed method involves the use of commercially available resins to sequester nitrate and phosphorus in a tube-like sampler called SorbiCell. This sampler has recently been proved to be a cost-effective tool to estimate reliable average concentrations of nitrate and phosphorus in a water catchment (Rozemeijer et al., 2010).

\section{Diffusive gradients in thin-films (DGT)}

The DGT technique is initially developed to measure trace metal concentrations in natural waters and trace metal fluxes from solid phase to solution in sediments and soils (Davlson and Zhang, 1994). The technique is a further development of the passive sampling principle where a device can accumulate the compounds of interest on a binding agent after passage through a hydrogel which acts as a well defined diffusion layer (Davlson and Zhang, 1994). It relies on the establishment of a steadystate concentration gradient from solution to the binding agent. Concentration in solution is calculated using Fick's first law of diffusion and the measured mass of solutes accumulated on the binding agent after a known deployment time as mentioned in the previous sections. The concentration gradient of phosphorus through the DGT sampler is represented in Fig. 1.

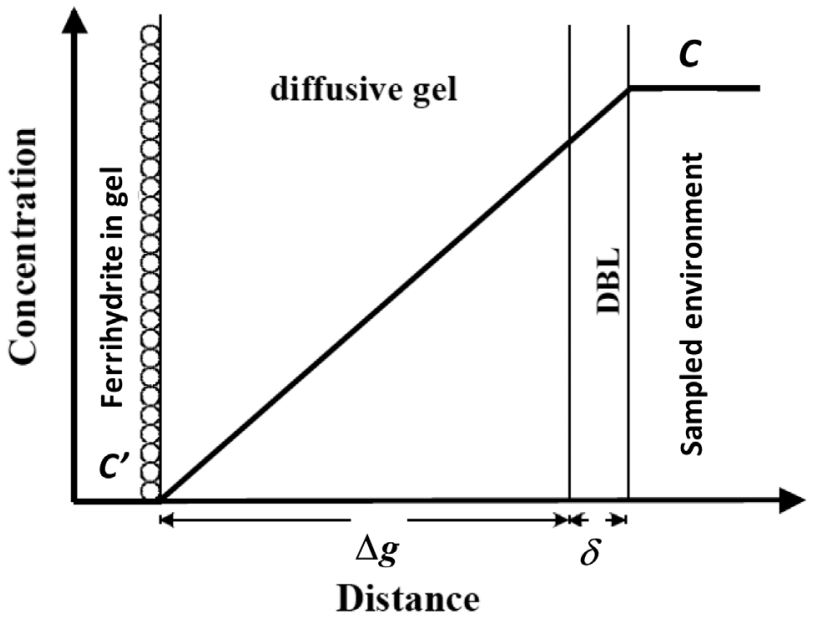

Fig. 1. The concept of concentration gradient (of phosphorus) through a DGT passive sampling device, $C, C^{\prime}$ are the concentrations of phosphorus in the environment and in the binding agent, $\Delta g$ and $\delta$ are the thicknesses of the gel and the diffusion layer, respectively (Zhang et al. (1998)).

The detailed description of the device for phosphorus can be found in Zhang et al. (1998). For measurement of reactive phosphorus species, a layer of gel impregnated with ferrihydrite (oxide-gel) was used as binding agent. It was constantly separated from the sampled environment by an ion-permeable gel layer of thickness $\Delta g$ as shown in Fig. 1. The gel layer is kept within the sampler by a filter membrane that allows free transport of target compounds. As in the principle of other passive samplers, there is usually a diffusive boundary layer (DBL), of $\delta$ thickness, where transport of ions is solely by molecular diffusion between the membrane and the sampled environment. If $\delta$ is negligible compared to $\Delta g$ then according the Fick's law the flux (F) of phosphorus species diffused through the gel layer to the ferrihydrite gel can be expressed by Eq. (3).

$$
F=\frac{D\left(C-C^{\prime}\right)}{\Delta g}
$$


in which $D$ is the effective diffusion coefficient of phosphorus species in the gel, $C$ and $C^{\prime}$ are concentration of phosphorus in different compartments as shown in Fig. 1.

Because ferrihydrite can be considered as a sink for phosphorus, $C^{\prime}$ is considered close to zero. Using the definition of flux, $F=M / A t$, with $M$ is the mass of phosphorus diffused through the area $A$ of the sampler during a time $t$, Eq. (3) can be rearranged to be an Eq to estimate the concentration of phosphorus in the sampled environment (Eq. (4)).

$$
C=\frac{M \Delta g}{D A t}
$$

As a consequence, if the mass of phosphorus sequestered by a passive sampler can be measured accurately, one can estimate the time-average concentration of phosphorus in this compartment using a pre-calibrated passive sampler (known diffusion coefficient)

The design and actual DGT passive sampler for water are shown in Fig. 2. More detailed information about the development and validation of the DGT sampler for phosphorus can be found in (Zhang et al., 1998).
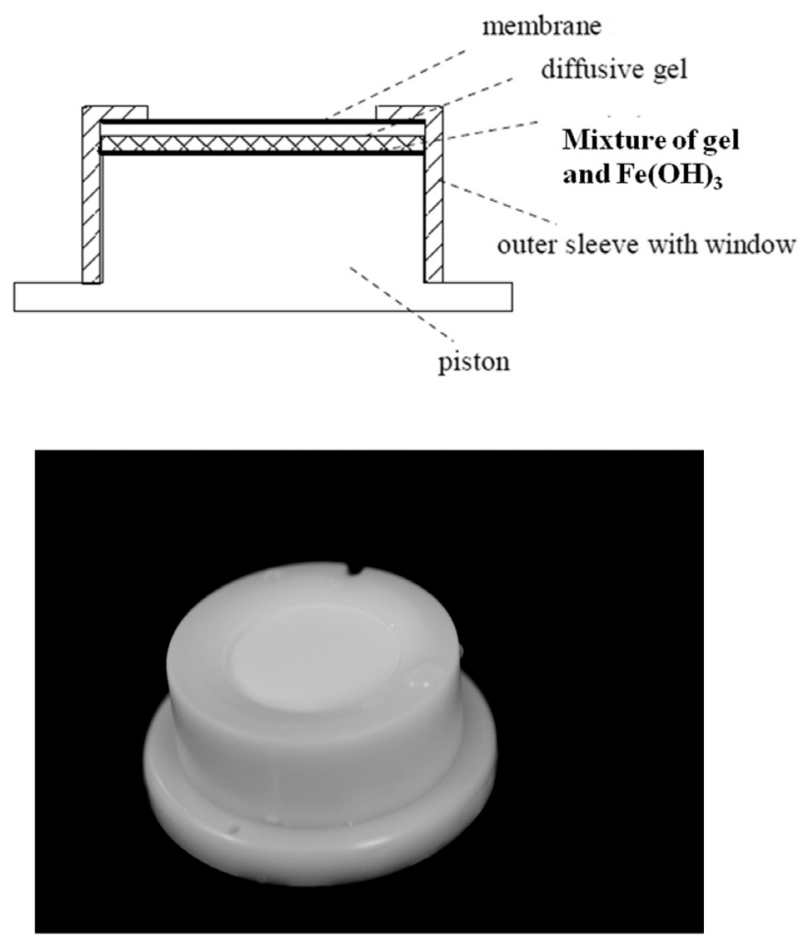

Fig. 2. Design (cross-section) and an actual DGT passive sampler (Adapted from DGT Research Ltd website).

Currently, the DGT samplers are manufactured in standard forms and are commercially available for measuring phosphorus in both water and sediment. More information about the products can be provided by DGT Research Ltd (http://www.dgtresearch.com/DGTResearch Tech.html).

\section{P-trap samplers}

This sampler shares the same principle with the DGT sampler which uses Ferrihydrite as a binding agent to attract phosphates from the sampled environment.
However, the preparation of the sampler is simplified to reduce cost associated with each sampler deployment.

The main purpose of $\mathrm{P}$-trap is to provide a simple, easy to handle, and cost effective method for the estimation of dissolved reactive phosphorus loads in streams and rivers (Müller et al., 2007). Utilizing Eq. (1) the estimation of phosphorus load will be based on (1) continuous recording of the water level (discharge) and (2) average concentration of phosphorus obtained from passive samplers (P-trap) deployed/exposed in the streams or rivers of interest.

The P-trap sampler only requires ferrihydrite which settles naturally on the surface of a membrane by inversely turn a bottle containing this oxide in water upside-down (Müller et al., 2007). This simplification led to a change in the diffusion principle of this sampler (Fig. 3). However, experiments have proved that the uptake of phosphorus is still effective with linear correlation with the exposure doses (Müller et al., 2007)

Initially, the P-trap is designed to trap phosphorus in a single cavity as shown in Fig. 3. However, as the authors recognize the effect of flow/discharge on the concentration of phosphorus where high flow usually accompanies
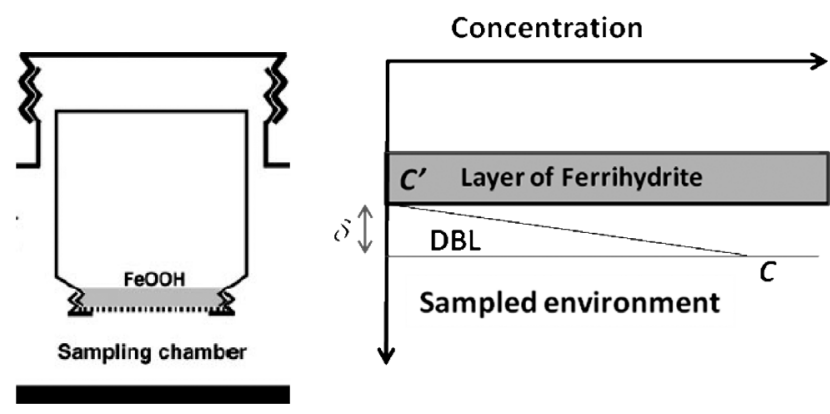

Fig. 3. The design of the first P-trap (Müller et al. (2007)) and its corresponding diffusion principle.

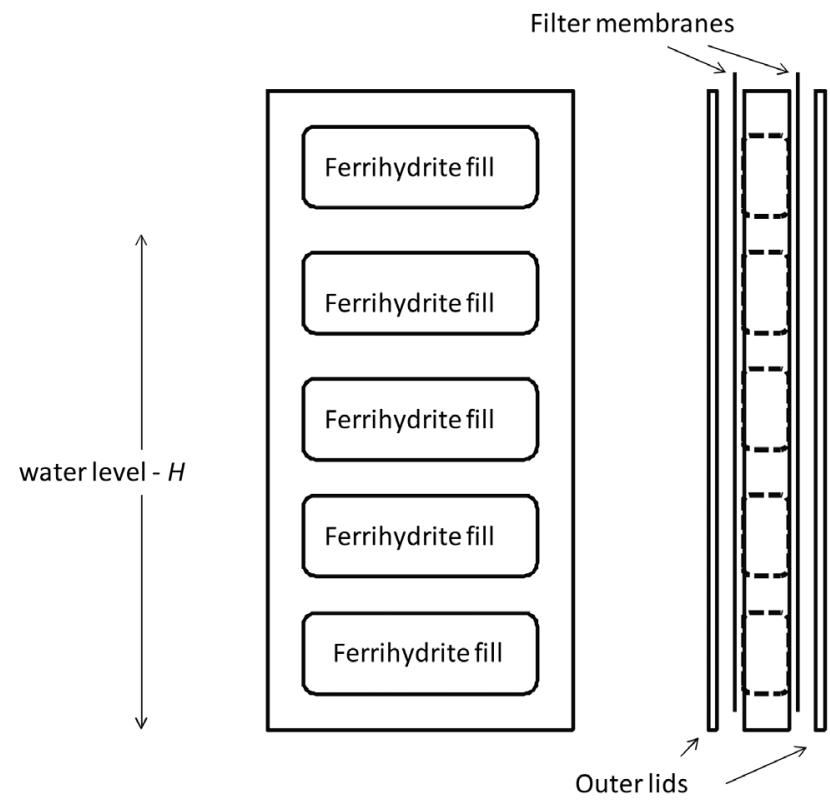

Fig. 4. Improved P-trap for measuring phosphorus in stream with variable water level (Adapted from Muller et al. (2008)). 
high concentration thanks to wash-off effect a multi-level sampler was proposed. The new designed was based on the sampler used for sediment and was shown in Fig. 4 (Muller et al., 2008). The individual sampler compartment will only sequester phosphorus when it is immersed in the stream. As a consequence, the amount of phosphorus $(\mathrm{P})$ in each compartment is proportional to the average concentration of phosphorus in the stream during its immersion period. With a system of multi-level sampler and a automatic water level logger one has a more accurate estimate of phosphorus concentrations in the stream including the effect of flow to the concentration. Detailed discussion about this new sampler can be found elsewhere (Muller et al., 2008).

\section{SorbiCell samplers}

The principle of this sampler was first proposed to measure mobile solutes in general in soil and groundwater (de Jonge and Rothenberg, 2005). Depending on the selected sorbent used in the cell, the sampler can cover a wide range of solutes including pesticides, phosphates, and hydrophobic organic contaminants (HOCs).

SorbiCell consists of two key compartments. The first compartment is a water-permeable porous sampler that is in continuous capillary contact with its surroundings and functions as a semi-infinite adsorptive sink for the solutes of interest. The second compartment contains a tracer that can be dissolved and release proportional to the amount of water going through the sampler. The amount of tracer left at the time of collection will provide information on the volume of water that has passed the sampler during the deployment period. The advantage of this new method is that it can account for concentration dynamics, because it gives a flow-proportional weighted average that is representative of the entire sampling period (de Jonge and Rothenberg, 2005).

The design of the SorbiCell sampler is shown in Fig. 5 where solutes, e.g. phosphorus compounds will go into contact with the sorbent and will be retained in this sorbent compartment. Meanwhile, the flow through will dissolve part of the tracer and take it away from the tracer compartment. The latest sampler was pictured to have the form of a commercially available SPE cartridge and was tested successfully for the ability to measure nitrate and phosphorus in a catchment in the Netherlands (Rozemeijer et al., 2010).

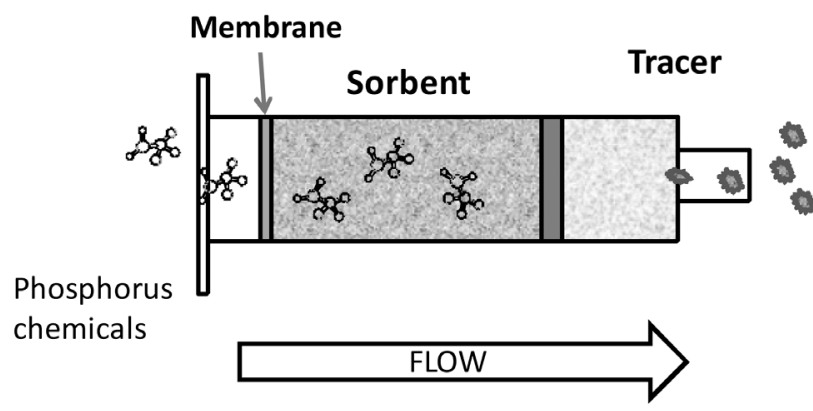

Fig. 5. Design and the mechanism of SorbiCell passive sampler (Adapted from Rozemeijet et al. (2010)).
To estimate the load using a SorbiCell, one has to measure both the mass of phosphorus adsorbed in the sorbent compartment (MP) and the mass of remaining tracer $\left(M_{T r, t}\right)$. Then the volume of water passing through the sampler is calculated as:

$$
\mathrm{V}=\frac{M_{t, 0}-M_{t}}{C_{t, \max }}
$$

The average concentration of phosphorus in the flowing-through water flux (CP) is calculated as

$$
C_{P}=\frac{M_{P}}{V}
$$

It should be noted that care should be taken to avoid the complete dissolution of the tracer and the remaining amount should be from $20 \%-80 \%$ to ensure the accuracy of the volume estimation. It is also suggested to use a combination of tracer salts with different $K_{s p}$ values and/or different amounts to reduce the uncertainty and increase the range of measurement (de Jonge and Rothenberg, 2005).

\section{APPLICATION OF PHOSPHORUS PASSIVE SAMPLERS}

\section{For water quality monitoring}

Monitoring phosphorus in the aquatic environment is very important since in recent years it has become apparent that the concomitant increase in losses of $\mathrm{N}$ and $\mathrm{P}$ from agricultural land is having a serious detrimental effect on water quality and the environment (Hart et al., 2004). Many management practices and protection measures have been proposed in order to reduce the load of $\mathrm{P}$ in the environment. To evaluate the effectiveness of such measures, continuous monitoring of water bodies is critical. However, most of monitoring studies about phosphorus in the aquatic environment have used grab samples which may compromise the representativeness of the phosphorus load estimation. O'Brien et al. (2011) has used a passive flow monitor (PFM) device based on gypsum dissolution rates as a complimentary tool for the DGT-like sampler. The PFMs will provide an estimated flow of water in the sampling site and thus help improve the quantification of phosphorus concentration. The recent study that use passive sampling technique for phosphorus is in the Netherlands (Rozemeijer et al., 2010) where SorbiCell samplers were deployed in a medium size catchment to measure phosphorus in water and sediment. However, as mentioned in the previous paper (Phong et al., 2012), the passive sampling techniques although very promising still have a long way toward being accepted as a conventional method.

\section{For soil/sediment quality monitoring}

Two P-passive samplers described above have designs for soil/sediment matrix, the DGT and the SorbiCell (de Jonge and Rothenberg, 2005, Ding et al., 2012). This design would help the monitoring of phosphorus in soil water. SorbiCell design was tested in soil up to the depth of $20 \mathrm{~cm}$ with 2 or $4 \mathrm{~cm}$ increments (de 
Jonge and Rothenberg, 2005). SorbiCell can also provide the additional information of flow in the sampling soil/ sediment. The commercial DGT sampler is also available for soil/sediment matrix in form of sampling plates. And those samplers were used to establish a novel approach to measure the phosphorus concentration in surface runoff (Dougherty et al., 2011). The DGT technique was also put into test with other conventional methods for phosphorus in soil (Six et al., 2012). The authors concluded that most conventional soil tests can extract a fraction of phosphorus which is not available to maize. The DGT technique, however, only samples P from the plant-accessible pool. Meanwhile, Ding et al. (2012) has further developed the DGT technique for the high-resolution simultaneous measurements of dissolved reactive phosphorus and dissolved sulfide in sediment and has achieved a good performance.

The heterogeneity of soil/sediment matrix means that measurement of each sampler only provides point data. However, it also makes the use of passive sampling an advantage because it can reduce considerably the cost of any sampling campaign for the spatial distribution of phosphorus concentration in soil/sediment.

\section{Applications of these techniques in Japan}

In the previous paper, we have reviewed the use of passive sampling techniques in Japan in general as well as in agricultural area. The conclusion is that the use of passive sampling in Japan still very limited. Therefore, it is understandable that no result from the search in Web of Knowledge ${ }^{\circledR}$ about the use of passive sampling for phosphorus in Japan. However, there are studies which applied the DGT techniques to measure metal ions in aquatic environment (Nyein Aung et al., 2008) or very recently to assess bioavailability of metal ions in river water (Naito et al., 2011). Again it is suggested that the advantage of passive sampling technique in environmental monitoring should be utilized in future research in Japan.

\section{REFERENCES}

Aung, N. N., F. Nakajima and H. Furumai 2008 Trace metal speciation during dry and wet weather flows in the Tama River, Japan, by using diffusive gradients in thin films DGT. Journal of Environmental Monitoring, 10: 219-230

Davlson, W. and H. Zhang 1994 In situ speciation measurements of trace components in natural waters using thin-film gels. Nature, 367: 546-548

de Jonge, H. and G. Rothenberg 2005 New device and method for Flux-proportional sampling of mobile solutes in soil and groundwater. Environmental Science \& Technology, 39: 274-282

Ding, S., Q. Sun, D. Xu, F. Jia, X. He and C. Zhang 2012 Highresolution simultaneous measurements of dissolved reactive phosphorus and dissolved sulfide: The first observation of their simultaneous release in sediments. Environmental Science and Technology, 46: 8297-8304

Dougherty, W. J., S. D. Mason, L. L. Burkitt and P. J. Milham 2011 Relationship between phosphorus concentration in surface runoff and a novel soil phosphorus test procedure -DGTunder simulated rainfall. Soil Research, 49: 523-528

Górecki, T. and J. Namieśnik 2002 Passive sampling. TrAC Trends in Analytical Chemistry, 21: 276-291

Hart, M. R., B. F. Quin and M. L. Nguyen 2004 Phosphorus runoff from agricultural land and direct fertilizer effects. Journal of Environmental Quality, 33: 1954-1972

Kot-Wasik, A., B. Zabiegała, M. Urbanowicz, E. Dominiak, A. Wasik and J. Namieśnik 2007 Advances in passive sampling in environmental studies. Analytica Chimica Acta, 602: 141-163

Mason, S., R. Hamon, H. Zhang and J. Anderson 2008 Investigating chemical constraints to the measurement of phosphorus in soils using diffusive gradients in thin films DGT and resin methods. Talanta, 74: 779-787

Müller, B., A. Stöckli, R. Stierli, E. Butscher and R. Gächter 2007 A low cost method to estimate dissolved reactive phosphorus loads of rivers and streams. Journal of Environmental Monitoring, 9: 82-86

Muller, B., R. Stierli and R. Gachter 2008 A low-tech, low-cost passive sampler for the long-term monitoring of phosphate loads in rivers and streams. Journal of Environmental Monitoring, 10: 817-820

Naito, W., M. Mori, Y. Iwasaki, M. Kamo and S. Musunaga 2011 Measurement of bioavailability of metals in river water using DGT. Journal of Japan Society on Water Environment, 34: $65-71$

O’Brien, D. S., K. Booij, D. W. Hawker and J. F. Mueller 2011 Method for the in situ calibration of a passive phosphate sampler in estuarine and marine waters. Environmental Science and Technology, 45: 2871-2877

Panther, J. G., P. R. Teasdale, W. W. Bennett, D. T. Welsh and H. Zhao, 2010 Titanium dioxide-based DGT technique for in situ measurement of dissolved reactive phosphorus in fresh and marine waters. Environmental Science and Technology, 44 9419-9424

Phong, T. K., D. H. Phuc, L. A. Tuan, T. H. Phi and K. Hiramatsu 2012 Applications of passive sampling techniques in monitoring organic pollutants in the environment. Journal of the Faculty of Agriculture, Kyushu University, 57: 169-174

Pichette, C., H. Zhang and S. Sauvé 2009 Using diffusive gradients in thin-films for in situ monitoring of dissolved phosphate emissions from freshwater aquaculture. Aquaculture, $\mathbf{2 8 6}$ : 198-202

Rozemeijer, J., Y. van der Velde, H. de Jonge, F. van Geer, H.-P. Broers and M. Bierkens 2010 Application and evaluation of a new passive sampler for measuring average solute concentrations in a catchment scale water quality monitoring study. Environmental Science \& Technology, 44: 1353-1359

Six, L., P. Pypers, F. Degryse, E. Smolders and R. Merckx 2012 The performance of DGT versus conventional soil phosphorus tests in tropical soils - An isotope dilution study. Plant and Soil, 359: 267-279

Stockdale, A., W. Davison and H. Zhang 2008 High-resolution two-dimensional quantitative analysis of phosphorus, vanadium and arsenic, and qualitative analysis of sulfide, in freshwater sediment. Environmental Chemistry, 5:143-9

Zhang, H., W. Davison, R. Gadi and T. Kobayashi 1998 In situ measurement of dissolved phosphorus in natural waters using DGT. Analytica Chimica Acta, 370: 29-38 